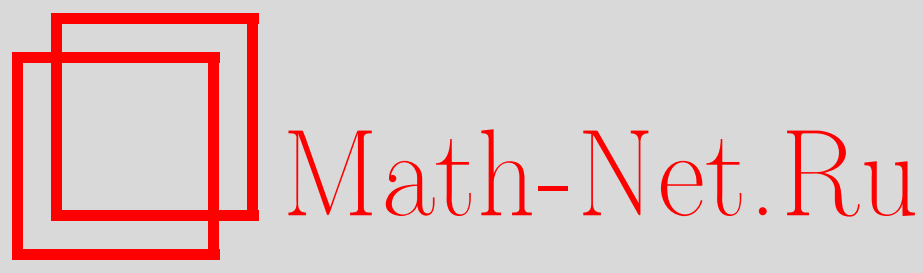

В. Р. Халилов, Спонтанное рождение фермионов в кулоновском поле и в потенциале Ааронова-Бома в $2+1$ измерениях, ТМФ, 2009, том 158, номер 2, 250-262

DOI: https://doi.org/10.4213/tmf6312

Использование Общероссийского математического портала Math-Net.Ru подразумевает, что вы прочитали и согласны с пользовательским соглашением http://www . mathnet.ru/rus/agreement

Параметры загрузки:

IP : 18.208 .226 .222

26 апреля 2023 г., 13:32:26

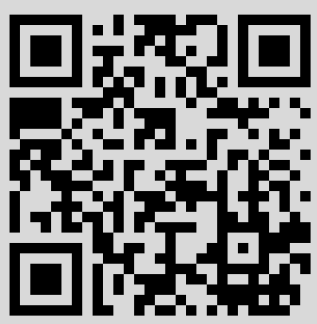




\section{СПОНТАННОЕ РОЖДЕНИЕ ФЕРМИОНОВ В КУЛОНОВСКОМ ПОЛЕ И В ПОТЕНЦИАЛЕ ААРОНОВА-БОМА В $2+1$ ИЗМЕРЕНИЯХ}

Найдены точные решения уравнения Дирака и спектр энергий фермиона в кулоновских (векторном и скалярном) полях и в потенциале Ааронова-Бома с учетом спина частицы в $2+1$ измерениях. Для описания спина фермиона используется двухкомпонентное уравнение Дирака с дополнительным (спиновым) параметром, введенным Хагеном. Рассмотрен эффект рождения пар фермионов из вакуума сильным кулоновским полем в потенциале Ааронова-Бома в $2+1$ измерениях. Получены трансцендентные уравнения, которые неявно определяют спектр энергий электрона вблизи границы нижнего континуума энергий и критический заряд. Уравнение для критического заряда решено численно. Показано, что для относительно слабых магнитных потоков критический заряд уменьшается (по сравнению со случаем отсутствия магнитного потока), если энергия взаимодействия спинового магнитного момента электрона с магнитным полем отрицательна, и увеличивается, если эта энергия положительна.

Ключевые слова: спин, кулоновское поле, потенциал Ааронова-Бома, связанное состояние, спектр энергий, нижний континуум, критический заряд, рождение пар.

\section{1. ВВЕДЕНИЕ}

Эффект Ааронова-Бома, предсказанный в работе [1], - одно из наиболее интересных и интригующих явлений квантовой механики. Этот эффект был разносторонне изучен в многочисленных работах (см., например, книгу [2] и ссылки в ней). Он возникает при движении электронов в поле векторного потенциала $A_{\mu}$, магнитный поток которого заключен в цилиндрическую трубку исчезающе малого радиуса.

В цилиндрически-симметричной конфигурации внешнего поля, когда естественно предположить инвариантность квантово-механической системы относительно поворотов вокруг оси симметрии поля (оси $z$ ), изучаемая система становится существенно двумерной в плоскости $x y[3]$. Целый ряд физических явлений, имеющих место

*Московский государственный университет им. М.В. Ломоносова, Москва, Россия. E-mail: khalilov@phys.msu.ru 
в квантовых системах заряженных фермионов в $3+1$ измерениях, которые обладают аксиальной симметрией, более полно можно исследовать с помощью соответствующего уравнения Дирака в $2+1$ измерениях. Эффективность такой редукции к $2+1$ измерениям связана с возможностью получения точных решений уравнения Дирака в $2+1$ измерениях в виде специальных функций в более широком (по сравнению со случаем $3+1$ измерений) классе внешних полей.

Точные решения уравнения Дирака в $2+1$ измерениях в потенциале АароноваБома впервые были получены в работе [4], где, в частности, был рассмотрен процесс рождения частиц нестатическим полем движущейся космической струны. В работе [5] впервые были построены самосопряженные (на всей плоскости) гамильтонианы для квантово-механических задач рассеяния электронов тонкими соленоидами бесконечной и полубесконечной $(0, \infty)$ длины и найдены решения соответствующих уравнений Шредингера.

Точные решения уравнения Дирака и спектр энергий фермиона в $2+1$ измерениях в двумерных кулоновских (векторном и скалярном) полях и в потенциале Ааронова-Бома были найдены в работе [6], что позволило изучить влияние потенциала Ааронова-Бома на связанные состояния электрона в кулоновских полях, когда движение электрона становится финитным, а физически наблюдаемой (калибровочно-инвариантной) величина есть энергия электрона в связанном состоянии. Это релятивистский аналог эффекта Ааронова-Бома для связанных состояний. Заметим, что нерелятивистский электрон, движущийся в замкнутом кольце в потенциале Ааронова-Бома, является связанным даже в отсутствие кулоновского поля.

Решения уравнения Дирака в $2+1$ измерениях не описывают две проекции спина фермиона, так как они представляют собой спинор, верхняя и нижняя компоненты которого интерпретируются, соответственно, как положительно- и отрицательно-частотные решения. Вместе с этим решения уравнения Дирака в $2+1$ измерениях позволяют изучить релятивистские эффекты.

Для описания спина в задаче рассеяния электронов потенциалом Ааронова-Бома в работе [7] в двухкомпонентное уравнение Дирака был введен спиновый параметр, и полученные решения были использованы для вычисления амплитуды и поперечного сечения рассеяния.

Задача рассеяния спин-поляризованных электронов векторным потенциалом Ааронова-Бома, который создает магнитный поток, заключенный в цилиндрическую трубку малого радиуса, была решена в работе [8] как с помощью решений уравнения Паули в $3+1$ измерениях, которое содержит член, описывающий взаимодействие трехмерного спинового магнитного момента электрона с магнитным полем трубки, так и с использованием решений уравнения Дирака в $2+1$ измерениях с новым параметром. В работе [9] аналогичная задача была решена в рамках квазиклассической модели в $3+1$ измерениях.

В настоящей статье мы изучаем влияние спина частицы на релятивистские связанные состояния фермиона в двумерных кулоновских (векторном и скалярном) полях и в потенциале Ааронова-Бома. Использование модели, описываемой уравнением Дирака в $2+1$ измерениях в указанной конфигурации полей, продиктовано 
тем, что только в $2+1$ измерениях удается исследовать влияние спина частицы и магнитного потока на устойчивость глубоких (релятивистских) связанных состояний, а также обусловленный неустойчивостью этих состояний процесс рождения позитронов из вакуума сильным кулоновским полем. Вместе с этим нелишне отметить, что двумерное кулоновское поле реализуется в двумерных кулоновских кристаллах [10].

В статье используется система единиц, в которой $c=\hbar=1$.

\section{2. РЕШЕНИЯ УРАВНЕНИЯ ДИРАКА В $2+1$ ИЗМЕРЕНИЯХ И СПЕКТР ЭНЕРГИЙ ФЕРМИОНА}

Уравнение Дирака в $2+1$ измерениях для фермиона массы $m$ и заряда $e=-e_{0}<0$ в векторном $A_{\mu}$ и скалярном $U$ потенциалах имеет вид

$$
\left(\gamma^{\mu} P_{\mu}-m-U\right) \Psi=0,
$$

где $P_{\mu}=-i \partial_{\mu}-e A_{\mu}$ - оператор обобщенного момента фермиона, а матрицы Дирака $\gamma^{\mu}$ удобно определить через двумерные матрицы Паули как

$$
\gamma^{0}=\sigma_{3}, \quad \gamma^{1}=i s \sigma_{1}, \quad \gamma^{2}=i \sigma_{2} .
$$

Здесь параметр $s= \pm 1$ характеризует два значения проекции спина электрона [7].

Найдем решения уравнения Дирака с учетом параметра $s$ в конфигурации потенциала Ааронова-Бома

$$
A^{0}=0, \quad A_{x}=-\frac{B y}{r^{2}}, \quad A_{y}=\frac{B x}{r^{2}} \quad \Leftrightarrow \quad A^{0}=0, \quad A_{r}=0, \quad A_{\varphi}=\frac{B}{r},
$$

где $r=\sqrt{x^{2}+y^{2}}, \varphi=\operatorname{arctg}(y / x)$, и векторного $A^{\mu}(r)$ и скалярного $U(r)$ потенциалов, заданных следующим образом:

$$
A^{0}(r)=\frac{a}{e_{0} r}, \quad A_{r}=0, \quad A_{\varphi}=0 ; \quad U(r)=-\frac{b}{r} \quad(a, b>0) .
$$

Оператор Гамильтона для рассматриваемой системы есть

$$
H_{\mathrm{D}}=\sigma_{1} P_{2}-s \sigma_{2} P_{1}+\sigma_{3}(m+U(r))-e_{0} A_{0}(r),
$$

а векторный потенциал $A_{\mu}$ в обобщенном импульсе $P_{\mu}$ есть сумма $(3)$ и (4). Собственные значения оператора полного углового момента $J_{z} \equiv L_{z}+s \sigma_{3} / 2$, где $L_{z} \equiv$ $-i \partial / \partial \varphi$, являются сохраняющимися величинами.

Положительно-частотные решения уравнения (1) с потенциалами (3) и (4) будем искать в виде (см. также [11]-[13])

$$
\Psi(t, \mathbf{x})=\frac{1}{\sqrt{2 \pi}} e^{-i(E t-l \varphi)} \psi(r, \varphi),
$$

где $E \geqslant 0$ - энергия фермиона, $l$ - целое число, а $\psi(r, \varphi)$ - двухкомпонентная функция (т.е. спинор),

$$
\psi(r, \varphi)=\left(\begin{array}{c}
f(r) \\
g(r) e^{i s \varphi}
\end{array}\right)
$$


Функции $f(r)$ и $g(r)$ нормированы условием

$$
\int_{0}^{\infty}\left(\left|f^{2}\right|+\left|g^{2}\right|\right) r d r=1 .
$$

Очевидно, волновая функция $\Psi$ является собственной функцией оператора полного углового момента $J_{z}$ с собственным значением $j=l+s / 2$.

Радиальные функции $f(r)$ и $g(r)$ удовлетворяют системе уравнений

$$
\begin{array}{r}
s \frac{d f}{d r}-\frac{l+e_{0} B}{r} f+\left(E+m+\frac{a-b}{r}\right) g=0, \\
s \frac{d g}{d r}+\frac{l+s+e_{0} B}{r} g-\left(E-m+\frac{a+b}{r}\right) f=0 .
\end{array}
$$

Решения этой системы уравнений будем искать в виде (см., например, [6], [12], [14])

$$
\begin{aligned}
& f(r)=\sqrt{m+E} e^{-x / 2} x^{\gamma_{s}}\left(Q_{1}+Q_{2}\right), \\
& g(r)=\sqrt{m-E} e^{-x / 2} x^{\gamma_{s}}\left(Q_{1}-Q_{2}\right),
\end{aligned}
$$

где

$$
x=2 \lambda r, \quad \lambda=\sqrt{m^{2}-E^{2}},
$$

а величина

$$
\gamma_{s}=-\frac{1}{2} \pm \sqrt{\left(l+e_{0} B+\frac{s}{2}\right)^{2}-a^{2}+b^{2}}
$$

определяет асимптотическое поведение волновой функции при малых $r$.

Отметим, что если $e_{0} B=N$, где $N$ - целое число, то полный магнитный поток $\Phi=2 \pi B$ квантован и $\Phi=\Phi_{0} N$, где $\Phi_{0} \equiv 2 \pi / e_{0}$ - элементарная единица магнитного потока, "флюксоид". Из формулы (12) видно, что при $a^{2}<\left(l+e_{0} B+s / 2\right)^{2}+b^{2}$ величина $\gamma_{s}$ вещественна. Чтобы обеспечить выполнение условия нормировки (8), $\gamma_{s}$ должна быть положительной. Если $a^{2}>\left(l+e_{0} B+s / 2\right)^{2}+b^{2}$, то $\gamma_{s}$ является комплексной величиной, а соответствующая волновая функция осциллирует при $r \rightarrow 0$ (парадокс Клейна [14], [15]). Выберем

$$
\gamma_{s}=-\frac{1}{2}+\sqrt{\left(l+e_{0} B+\frac{s}{2}\right)^{2}-a^{2}+b^{2}} .
$$

Подставляя (10) в (9), нетрудно получить уравнения

$$
\begin{aligned}
s x \frac{d Q_{1}}{d x}+ & {\left[s\left(\gamma_{s}+\frac{1}{2}\right)+\frac{x}{2}(1-s)-\frac{a E+m b}{\lambda}\right] Q_{1}+} \\
+ & {\left[-\left(l+e_{0} B+\frac{s}{2}\right)-\frac{a m+b E}{\lambda}\right] Q_{2}=0, } \\
s x \frac{d Q_{2}}{d x}+ & {\left[s\left(\gamma_{s}+\frac{1}{2}\right)-\frac{x}{2}(1+s)+\frac{a E+m b}{\lambda}\right] Q_{2}+} \\
+ & {\left[-\left(l+e_{0} B+\frac{s}{2}\right)+\frac{a m+b E}{\lambda}\right] Q_{1}=0 . }
\end{aligned}
$$


Исключая $Q_{1}$ или $Q_{2}$ из $(14)$, получим независимые уравнения второго порядка

$$
x \frac{d^{2} Q_{1,2}^{s}}{d x^{2}}+\left[2\left(\gamma_{s}+1\right)-x\right] \frac{d Q_{1,2}^{s}}{d x}-\left(\gamma_{s}+1 \mp \frac{s}{2}-\frac{a E+b m}{\lambda}\right) Q_{1,2}^{s}=0,
$$

где верхний (нижний) знак следует поставить для $Q_{1}^{s}\left(Q_{2}^{s}\right)$. Отметим также, что при получении этих уравнений мы использовали легко проверяемое равенство

$$
\left(\gamma_{s}+\frac{1}{2}\right)^{2}-\frac{a^{2}-b^{2}}{\lambda^{2}} E^{2}=\left(l+e_{0} B+\frac{s}{2}\right)^{2}-\frac{a^{2}-b^{2}}{\lambda^{2}} m^{2}
$$

Решения уравнения (15), конечные при $x=0$, можно выразить через вырожденную гипергеометрическую функцию $F\left(a_{1}, c_{1} ; x\right)$ :

$$
\begin{aligned}
& Q_{1}^{s}=A \cdot F\left(\gamma_{s}+1-\frac{s}{2}-\frac{a E+m b}{\lambda}, 2 \gamma_{s}+2 ; x\right), \\
& Q_{2}^{s}=C \cdot F\left(\gamma_{s}+1+\frac{s}{2}-\frac{a E+m b}{\lambda}, 2 \gamma_{s}+2 ; x\right) .
\end{aligned}
$$

Найдем связь между постоянными $A$ и $C$ :

$$
C=\frac{\left(s \gamma_{s}+s / 2\right)-(E a+m b) / \lambda}{l+e_{0} B+s / 2+(m a+b E) / \lambda} A .
$$

Волновая функция будет квадратично интегрируемой, если обе гипергеометрические функции $Q_{1}^{s}$ и $Q_{2}^{s}$ сводятся к полиномам. Для этого параметр $a_{1}$ функции $F\left(a_{1}, c_{1} ; x\right)$ должен быть целым отрицательным числом или нулем. Вводя обозначение

$$
-n_{r}=\gamma_{s}+1-\frac{s}{2}-\frac{E a+m b}{\lambda}
$$

для $s=1$ нетрудно показать, что если $n_{r}=1,2,3, \ldots$, то обе функции $Q_{1}^{1}$ и $Q_{2}^{1}$ сводятся к полиномам; если же $n_{r}=0$, то к полиномам сводится только $Q_{1}^{1}$. Но равенство $n_{r}=0$ подразумевает, что

$$
\gamma_{1}+\frac{1}{2}=\frac{E a+m b}{\lambda}>0
$$

а из (16) и (20) следует, что

$$
\frac{E b+m a}{\lambda}=\left|l+e_{0} B+\frac{1}{2}\right|
$$

Если $l+e_{0} B+1 / 2>0$, то $C=0$, поэтому $Q_{2}^{1}=0$, и необходимое условие не нарушается $\left(Q_{1}^{1}-\right.$ полином). Если $l+e_{0} B+1 / 2<0$, то $C=A$, а $Q_{2}^{1}$ остается растущей функцией. Таким образом, допустимы следующие значения $n_{r}: n_{r}=0,1,2, \ldots$ при $l+e_{0} B+1 / 2>0$ и $n_{r}=1,2,3, \ldots$ при $l+e_{0} B+1 / 2<0$.

Для $s=-1$ функции $Q_{1}^{-1}$ и $Q_{2}^{-1}$ сводятся к полиномам, если $n_{r}=0,1,2, \ldots$. Отметим, что для $s=-1$ квантовое число $n_{r}$ может принимать значение -1 . Если 
$n_{r}=-1$, то только одна функция $Q_{2}^{-1}$ сводится к полиному. Но при $n_{r}=-1$ из (19) находим, что

$$
\gamma_{-1}+\frac{1}{2}=\frac{E a+m b}{\lambda}>0,
$$

и равенство (21) приобретает вид

$$
\frac{E b+m a}{\lambda}=\left|l+e_{0} B-\frac{1}{2}\right|
$$

Полезно переписать равенство (18) для $s=-1$ как

$$
A=-\frac{l+e_{0} B-1 / 2+(m a+b E) / \lambda}{\left(\gamma_{-1}+1 / 2\right)+(E a+m b) / \lambda} C .
$$

Далее, если $l+e_{0} B-1 / 2<0$, то $A=0$, поэтому $Q_{1}^{-1}=0$, и необходимое условие не нарушается $\left(Q_{2}^{-1}-\right.$ полином). Если $l+e_{0} B-1 / 2>0$, то $A=-C$, а $Q_{1}^{-1}$ остается растущей функцией. Таким образом, для $s=-1$ допустимы следующие значения $n_{r}$ : $n_{r}=-1,0,1, \ldots$ при $l+e_{0} B-1 / 2<0$ и $n_{r}=0,1,2, \ldots$ при $l+e_{0} B-1 / 2>0$.

Преобразование $s \rightarrow-s$ приводит уравнение (15) к виду

$$
x \frac{d^{2} Q_{1,2}^{-s}}{d x^{2}}+\left[\left(2 \gamma_{-s}+1\right)-x\right] \frac{d Q_{1,2}^{-s}}{d x}-\left(\gamma_{-s}+1 \pm \frac{s}{2}-\frac{a E+b m}{\lambda}\right) Q_{1,2}^{-s}=0,
$$

где верхний (нижний) знак должен быть взят для $Q_{1}^{-s}\left(Q_{2}^{-s}\right)$. Конечные при $x=0$ решения (25) суть

$$
\begin{aligned}
& Q_{1}^{-s}=A \cdot F\left(\gamma_{-s}+1+\frac{s}{2}-\frac{a E+m b}{\lambda}, 2 \gamma_{-s}+2 ; x\right) \\
& Q_{2}^{-s}=C \cdot F\left(\gamma_{-s}+1-\frac{s}{2}-\frac{a E+m b}{\lambda}, 2 \gamma_{-s}+2 ; x\right),
\end{aligned}
$$

где константы $A$ и $C$ связаны равенством

$$
A=\frac{\left(s \gamma_{s}+s / 2\right)+(E a+m b) / \lambda}{l+e_{0} B+s / 2-(m a+b E) / \lambda} C .
$$

Повторяя рассуждения, приведенные после соотношения (18), естественным образом получим те же результаты для всех допустимых значений квантовых чисел.

Из соображений непрерывности состояния частиц - это состояния, которые при бесконечно медленном выключении внешнего поля примыкают к границе непрерывного спектра $E=m$ (см., например, [14]).

Переписав уравнение (19) как

$$
\frac{E a+m b}{\lambda}=n_{r}+\gamma_{s}+1-\frac{s}{2} \equiv v,
$$

найдем дискретные уровни энергии фермиона:

$$
\frac{E_{n}}{m}=\sqrt{\left(\frac{a b}{v^{2}+a^{2}}\right)^{2}+\frac{v^{2}-b^{2}}{v^{2}+a^{2}}}-\frac{a b}{v^{2}+a^{2}} .
$$


Напомним, что магнитный поток входит в $\gamma_{s}(13)$ и, следовательно, влияет на спектр энергий фермиона. Заметим, что когда магнитный поток равен целому числу в единицах $\Phi_{0}$, спектр энергий тот же, что и в отсутствие магнитного потока. Если магнитный поток не равен целому числу в единицах $\Phi_{0}$, зависимость спектра энергий от магнитного потока, вероятно, можно наблюдать в оптических экспериментах, например по излучению. Интересно, что и эффект Ааронова-Бома возникает только тогда, когда магнитный поток не равен целому числу в единицах $\Phi_{0}$. Если магнитный поток не равен целому или полуцелому числам в единицах $\Phi_{0}$, то все уровни энергии, за исключением уровня с $l=0, n_{r}=0, s=1$ при $e_{0} B>0$ и уровня с $l=0, n_{r}=-1, s=-1$ при $e_{0} B<0$, дважды вырожденны (для определенности мы рассматриваем случай $l \geqslant 0)$; уровни с $l, n_{r}+1, s=1$ и $l+1, n_{r}, s=-1$ совпадают. Это означает, что собственные значения энергии фермиона, за исключением низшего уровня, в рассматриваемой конфигурации полей, фактически, не зависят от спина.

В отсутствие скалярного потенциала спектр энергий определяется выражением

$$
E_{n, l}=m\left[1+\frac{a^{2}}{\left(n_{r}+1 / 2-s / 2+\sqrt{\left(l+e_{0} B+s / 2\right)^{2}-a^{2}}\right)^{2}}\right]^{-1 / 2} .
$$

Подчеркнем, что не существует квантовых состояний при любых значениях $a$, если магнитный поток точно равен полуцелому числу $-l-s / 2$ в единицах $\Phi_{0}$ (т.е. $e_{0} B=$ $-l-s / 2)$, так как величина $\gamma_{s}$, а с ней и параметр $a_{1}$ всех гипергеометрических функций становятся комплексными. Очевидно, формула (30) также имеет смысл только при $\left|l+e_{0} B+s / 2\right|>a$, что запрещает существование уровней энергии с $l+e_{0} B+s / 2=0$, так как формула (30) при $l+e_{0} B+s / 2=0$ дает комплексное значение, и интерпретация $E_{n, l}$ как энергии электрона теряет смысл.

В нерелятивистском пределе выражение для спектра энергий электрона принимает вид

$$
E_{\mathrm{non}}=-\frac{a^{2}}{2\left(n_{r}+1 / 2-s / 2+\left|l+e_{0} B+s / 2\right|\right)^{2}} .
$$

Заметим, что при полуцелых $e_{0} B$ спектр энергий (31) определяется только целыми числами, и, по-видимому, его можно наблюдать, используя методы спектроскопии. Интересно, что при полуцелых $e_{0} B$ поперечное сечение рассеяния электронов потенциалом Ааронова-Бома как функция $e_{0} B$ принимает максимальное значение. Наконец, видно, что любое собственное значение энергии фермиона в рассматриваемой конфигурации полей есть периодическая функция магнитного потока подобно случаю движения фермиона в потенциале Ааронова-Бома в замкнутом кольце в отсутствие кулоновских потенциалов (см. также [16]). Спектр энергий повторяет себя всякий раз, когда $e_{0} B$ изменяется на целое число.

В отсутствие векторного кулоновского потенциала низший уровень энергии задается формулой

$$
E_{0}=m \frac{\left|l+e_{0} B+s / 2\right|}{\sqrt{\left(l+e_{0} B+s / 2\right)^{2}+b^{2}}},
$$

из которой видно, что $E_{0} \rightarrow 0$ даже при $b \rightarrow \infty$. В этом случае система стабильна при любых $b$. 


\section{3. КРИТИЧЕСКИЙ ЗАРЯД. РОЖДЕНИЕ ПОЗИТРОНОВ}

Рассмотрим нашу квантовую систему при больших $a$, полагая для простоты $b=0$, другими словами, рассмотрим задачу о стабильности вакуума квантовой электродинамики в $2+1$ измерениях в сильном кулоновском поле в присутствии магнитного потока. Следует отметить, что в отсутствие потенциала Ааронова-Бома эта проблема была всесторонне изучена в работах [17]-[22] в $3+1$ измерениях и в работах [12], [13] в $2+1$ измерениях.

Из формулы (29) видно, что низшие электронные состояния в конфигурации кулоновского поля и потенциала Ааронова-Бома суть состояния с $n_{r}=l=0, s=1$ и $n_{r}=-1, l=0, s=-1$ и с энергией

$$
E_{s}(a)=m \sqrt{\frac{\left(e_{0} B+s / 2\right)^{2}-a^{2}}{\left(e_{0} B+s / 2\right)^{2}}} .
$$

Заметим следующее: если магнитный поток равен $1 / 2$ в единицах $\Phi_{0}$, то состояние с $n_{r}=-1, l=0, s=-1$ при любых значениях $a$ не существует, так как нарушается основное условие $(22)$, и параметр $a_{1}$ гипергеометрической функции $Q_{2}^{-1}\left(a_{1}, c_{1} ; x\right)$ становится чисто мнимым. Для магнитного потока, равного $-1 / 2$ в единицах $\Phi_{0}$, не существует состояния с $n_{r}=0, l=0, s=1$ при любых значениях $a$, так как теперь становится чисто мнимым параметр $a_{1}$ гипергеометрической функции $Q_{1}^{1}\left(a_{1}, c_{1} ; x\right)$. Для этих значений магнитных потоков величина (33) расходится и является чисто мнимой. Поэтому в дальнейшем, не ограничивая общности рассмотрения проблемы, для определенности будем считать магнитный поток положительным и не равным $1 / 2$.

Энергия $E_{s}(a)$ как функция $a$ обращается в нуль при $a=\left|e_{0} B+s / 2\right|$ и становится чисто мнимой при $a>\left|e_{0} B+s / 2\right|$, поэтому интерпретация формулы (33) как энергии электрона теряет смысл при $a \geqslant\left|e_{0} B+s / 2\right|$. Это не означает, что при $a \geqslant\left|e_{0} B+s / 2\right|$ уравнение для энергии не имеет вещественных решений. Для того чтобы получить уравнение для энергии, имеющее смысл при $a \geqslant\left|e_{0} B+s / 2\right|$, необходимо наложить некоторое граничное условие на волновую функцию в точке источника. Действительно, дираковский гамильтониан в сильном кулоновском поле точечного заряда (источника) (4) становится не эрмитовым в точке, где находится источник, и постановка граничного условия в этой точке, очевидно, эквивалентна определению соответствующего самосопряженного расширения этого оператора [23]. Чтобы получить уравнение для энергии электрона при $a \geqslant\left|e_{0} B+s / 2\right|$, мы поставим граничное условие на некотором расстоянии $R$ от источника. Физически постановка такого граничного условия означает обрезание кулоновского потенциала на малых расстояниях, т.е. учет конечных размеров источника поля. Такой способ определения самосопряженного гамильтониана называют физической регуляризацией [5].

Задача о поведении электрона в сильном (обрезанном) кулоновском поле в $2+1$ измерениях в отсутствие потенциала Ааронова-Бома была изучена в работах [12], [13]. В этих работах, в частности, было показано, что с увеличением $а$ низший уровень энергии электрона при $a^{2}>1 / 4$ становится отрицательным и при дальнейшем увеличении $а$ может достичь границы нижнего континуума (отрицательных)

4 Теоретическая и математическая физика, т. 158, № 2, 2009 г. 
энергий $-m$. Значение $a=a_{\mathrm{cr}}$, при котором низший уровень энергии электрона равен $-m$, называют критическим зарядом для основного состояния. При $a \geqslant a_{\text {cr }}$ низший уровень энергии электрона пересекает границу нижнего континуума энергий, вакуум квантовой электродинамики в $2+1$ измерениях в сильном кулоновском потенциале перестраивается и становится неустойчивым по отношению к рождению электрон-позитронных пар.

Из формулы (33) видно, что на величину критического заряда влияют также магнитный поток и спин частицы. Чтобы получить уравнение, определяющее критический заряд $a_{\mathrm{cr}}(B)$, рассмотрим решения уравнения Дирака вблизи границы нижнего континуума (отрицательных) энергий $-m$. Введем функции $F(r)=r f(r)$ и $G(r)=r g(r)$ и исключим $G(r)$ из $(9)$. В результате получим уравнение для функции $F(r)$ с $E \approx-m$ в виде

$$
\frac{d^{2} F}{d r^{2}}+\left(E^{2}-m^{2}+\frac{2 E a}{r}+\frac{a^{2}-\left(l+e_{0} B\right)\left(l+e_{0} B+s\right)}{r^{2}}\right) F=0 .
$$

Решение для $G(r)$ вблизи $E=-m$ можно найти из уравнения

$$
G(r)=\frac{l+e_{0} B+s}{a} F(r)-s \frac{r}{a} \frac{d F}{d r} .
$$

Решение уравнения (34), которое обращается в нуль при $r \rightarrow \infty$, можно выразить через функцию Уиттекера (см., например, [24]),

$$
F(r) \sim W_{\beta, i \theta}(2 \lambda r)
$$

где

$$
\beta=\frac{E a}{\lambda}, \quad \theta=\sqrt{a^{2}-\left(l+\frac{e_{0} B s}{2}\right)^{2}},
$$

если $E \approx-m$, или через функцию Макдональда мнимого индекса,

$$
F(r) \sim \sqrt{r} K_{2 i \theta}(\sqrt{8 m a r})
$$

если $E=-m$. Из (36) или (38) следует, что электрон в связанном состоянии с $E \approx-m$ локализован в пространстве. Такое поведение электрона легко объяснить, если уравнение (34) для радиальной волновой функции трактовать как уравнение Шредингера для частицы с энергией $\epsilon=\left(E^{2}-m^{2}\right) / 2 m$, находящейся в эффективном потенциале

$$
U_{\text {eff }}(r)=-\frac{2 E a}{m r}-\frac{a^{2}-\left(l+e_{0} B\right)\left(l+e_{0} B+s\right)}{2 m r^{2}} .
$$

Видно, что при $E \approx-m$ потенциал $U_{\text {eff }}(r)$ для основного состояния электрона с $l=0$ имеет форму широкого барьера. Поэтому на больших расстояниях от кулоновского центра электрон с отрицательной энергией $E \approx-m$ не притягивается к нему, а отталкивается [23]. Следует отметить, что высота и ширина потенциального барьера увеличиваются в присутствии магнитного потока, если $s=1$ и $e_{0} B>1$. 
Вид потенциала при $r<R$ мало влияет на результаты, поэтому вычисления проведем для наиболее простой модели, полагая

$$
A^{0}(r)=\frac{a}{|e| R}, \quad A_{r}=0, \quad A_{\varphi}=0
$$

в области $r \leqslant R$ и предполагая, что магнитный поток сконцентрирован в области радиуса $R_{\mathrm{b}} \gg R$, так что соответствующий потенциал $\mathbf{A}$ в полярных координатах $r, \varphi$ в области $r<R_{\mathrm{b}}$ задается формулами

$$
A^{0}=0, \quad A_{r}=0, \quad A_{\varphi}=\frac{1}{2} H r .
$$

В области $r>R_{\mathrm{b}}$ потенциал $\mathbf{A}$ - это потенциал Ааронова-Бома. Поэтому при $r<R_{\mathrm{b}}$ уравнение для функции $f(r)$ имеет вид

$$
\left(\frac{\partial^{2}}{\partial r^{2}}+\frac{1}{r} \frac{\partial}{\partial r}+\left(E-\frac{a}{R}\right)^{2}-m^{2}-\frac{l^{2}}{r^{2}}-\frac{\left(e_{0} H r\right)^{2}}{4}-e_{0} H(l+s)\right) f(r)=0 .
$$

Вблизи $E=-m$ это уравнение можно переписать как

$$
\left(\frac{\partial^{2}}{\partial r^{2}}+\frac{1}{r} \frac{\partial}{\partial r}+\frac{a(a+2 m R)}{R^{2}}-\frac{l^{2}}{r^{2}}-\frac{\left(e_{0} B r\right)^{2}}{R_{b}^{4}}-\frac{2 e_{0} B}{R_{b}^{2}}(l+s)\right) f(r)=0 .
$$

Видно, что для $a>e_{0} B R^{2} / R_{\mathrm{b}}^{2}$ можно опустить член $\left(e_{0} B r\right)^{2} / R_{\mathrm{b}}^{4}$, и решение $f(r)$, конечное в точке $r=0$, в области $r \leqslant R$ выражается через функцию Бесселя целого индекса $|l|$ :

$$
f(r) \sim J_{|l|}(c r)
$$

где

$$
c=\sqrt{\left(E+\frac{a}{R}\right)^{2}-m^{2}-\frac{2 e_{0} B}{R_{\mathrm{b}}^{2}}(l+s)} .
$$

Сшиваем решения внутри и вне круга радиуса $R$ при $r=R$,

$$
\left.\frac{g(r)}{f(r)}\right|_{r=R-0}=\left.\frac{G(r)}{F(r)}\right|_{r=R+0}
$$

учитываем, что параметр $R$ должен быть мал по сравнению с $1 / m$ и $a / m$ и что $E \approx-m$, получаем трансцендентные уравнения, которые неявно определяют спектр энергий электрона вблизи границы континуума отрицательных энергий $-m$ :

$$
c R \frac{J_{1}(c R)}{J_{0}(c R)}=1-\left.x \frac{W_{\beta, i \theta}^{\prime}(x)}{W_{\beta, i \theta}(x)}\right|_{x=2 \lambda R},
$$

а также критический заряд для основного состояния:

$$
2 a_{0} \frac{J_{1}\left(a_{0}\right)}{J_{0}\left(a_{0}\right)}=1-\left.z \frac{K_{i \nu}^{\prime}(z)}{K_{i \nu}(z)}\right|_{z=\sqrt{8 m a_{\mathrm{cr}} R}}
$$


(при фиксированном малом $R$ ), $l=0$. Здесь

$$
\begin{gathered}
a_{0}=\sqrt{a_{\mathrm{cr}}^{2}-2 e_{0} B s \frac{R^{2}}{R_{\mathrm{b}}^{2}}}, \quad \beta=-\frac{m a}{\lambda}, \\
\theta=\sqrt{a^{2}-\left(e_{0} B+\frac{s}{2}\right)^{2}}, \quad \nu=2 \sqrt{a_{\mathrm{cr}}^{2}-\left(e_{0} B+\frac{s}{2}\right)^{2}},
\end{gathered}
$$

а штрихи обозначают дифференцирование по аргументу $x$ функции Уиттекера в (47) или по аргументу $z$ функции Макдональда в (48).

Уравнения (47) и (48) можно решить численно. Результаты численного решения уравнения (48) при различных значениях параметров приведены в таблицах.

ТАБлицА 1. Значения $a_{\mathrm{cr}}$ для $R \ll R_{\mathrm{b}}$

\begin{tabular}{|l|l|c|c|c|c|c|}
\cline { 2 - 7 } \multicolumn{2}{c|}{} & $B=0$ & $e_{0} B=0.1$ & $e_{0} B=0.2$ & $e_{0} B=0.6$ & $e_{0} B=1.1$ \\
\hline$R m=0.02$ & $s=1$ & 0.78 & 0.84 & 0.90 & 1.15 & 1.40 \\
\hline$R m=0.02$ & $s=-1$ & 0.78 & 0.75 & 0.68 & 0.64 & 0.84 \\
\hline$R m=0.006$ & $s=1$ & 0.70 & 0.77 & 0.84 & & \\
\hline
\end{tabular}

ТАБлицА 2. Значения $a_{\text {cr }}$ для $R=R_{\mathrm{b}}$

\begin{tabular}{|l|c|c|c|c|}
\cline { 3 - 5 } \multicolumn{1}{c|}{} & $B=0$ & $e_{0} B=1$ & $e_{0} B=0.2$ \\
\hline$R m=0.02$ & $s=1$ & 0.78 & 0.88 & 0.94 \\
\hline$R m=0.006$ & $s=1$ & 0.70 & 0.81 & 0.88 \\
\hline
\end{tabular}

Видно, что критический заряд увеличивается (уменьшается) с увеличением $B$ при $e_{0} B<0.8$ для $s=1$ (для $s=-1$ ); критический заряд уменьшается с уменьшением $R m$. Следовательно, нестабильность вакуума квантовой электродинамики в сильном кулоновском поле в $2+1$ измерениях в присутствии слабого магнитного потока для $s=-1$ (для $s=1$ ) должна проявляться при меньшем (большем) критическом заряде, т.е. в случае, когда взаимодействие спинового магнитного момента электрона с магнитным полем имеет характер притяжения (отталкивания), по сравнению со случаем отсутствия магнитного потока.

Уравнение (47) можно упростить, если $R m \ll 1$. В этом случае аргумент функции Уиттекера мал, и мы имеем

$$
\begin{aligned}
W_{\beta, i \theta}(x) & =\frac{\Gamma(2 i \theta)}{\Gamma(1 / 2-\beta+i \theta)} x^{1 / 2-i \theta}+\frac{\Gamma(-2 i \theta)}{\Gamma(1 / 2-\beta-i \theta)} x^{1 / 2+i \theta}= \\
& =\frac{|\Gamma(2 i \theta)|}{|\Gamma(1 / 2-\beta+i \theta)|} 2 x^{1 / 2} \cos (\Phi(x)),
\end{aligned}
$$

где

$$
\Phi(x)=-\theta \ln x+\arg \Gamma(2 i \theta)-\arg \Gamma\left(\frac{1}{2}-\beta+i \theta\right) .
$$


Дифференцируя равенство (50) по $x$ и подставляя результат и само равенство (50) в (47), получим следующее уравнение, которое явно содержит квантовое число $n_{r}$ :

$$
-\theta \ln (2 \lambda R)+\arg \Gamma(2 i \theta)-\arg \Gamma\left(\frac{1}{2}-\beta+i \theta\right)=\operatorname{arctg} Y+\pi n_{r},
$$

где

$$
Y=\theta^{-1}\left(\frac{1}{2}-c R \frac{J_{1}(c R)}{J_{0}(c R)}\right) .
$$

Аналогично можно вывести уравнение для спектра энергий при любых значениях $l$.

Уравнение (52) при $a<a_{\text {cr }}$ неявно определяет собственные значения энергии электрона в связанных состояниях для $l=0$ в области $m>E>-m$. Можно показать, что это уравнение имеет вещественные решения только при $a<a_{\mathrm{cr}}$, однако если $a>a_{\mathrm{cr}}$, то уравнение (52) имеет формальное комплексное решение вида $E=E_{0}-i w$, где $E_{0}=-m-c_{1}\left(a-a_{\mathrm{cr}}\right), c_{1} \sim 1$ для низшего состояния. Если $\Delta a \equiv a-a_{\mathrm{cr}} \ll a_{\mathrm{cr}}$, то мнимая часть $w$ экспоненциально мала. В этом случае решение уравнения (52) можно найти, аналитически продолжая $E$ как функцию $a$ в область $a>a_{\mathrm{cr}}$.

Однако если $\Delta a \ll a_{\mathrm{cr}}$, то $w$ можно найти проще. Действительно, при $a>a_{\mathrm{cr}}$ соответствующее уравнение Дирака для электрона имеет только формальное решение с $E=E_{0}-i w$. Вместе с этим при $a>a_{\text {cr }}$ то же уравнение описывает и фермион массы $m$, заряда $e_{0}$ с энергией $E_{0}=m+c_{1}\left(a-a_{\mathrm{cr}}\right)$ (естественно называть эту частицу

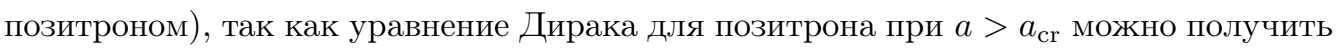
из уравнения Дирака для электрона заменой $E$ на $-E$ и $a$ на $-a$. При $a>a_{\text {cr }}$ квантовое состояние позитрона, очевидно, квазистационарно. Если $\Delta a \ll a_{\mathrm{cr}}$, то ширину квазистационарного уровня $w$ можно оценить в квазиклассическом приближении. Для этого мы должны вычислить коэффициент туннельного перехода частицы через потенциальный барьер. Величина $w$ определяет половину обратного времени жизни позитрона или удвоенную вероятность рождения электрон-позитронной пары кулоновским потенциалом. Если $\Delta a \ll a_{\mathrm{cr}}$, то кинетическая энергия рожденного позитрона мала, а кулоновский барьер очень широк и малопрозрачен. Следовательно, в этом случае вероятность рождения электрон-позитронной пары кулоновским потенциалом экспоненциально мала, т.е.

$$
w \sim m \exp \left[-2 \pi a\left(\frac{m}{\sqrt{E^{2}-m^{2}}}-1\right)\right] \cong m \exp \left(-c_{2} \sqrt{\frac{a_{\mathrm{cr}}}{\Delta a}}\right), \quad c_{2} \sim 1 .
$$

\section{4. ЗАКЛЮЧЕНИЕ}

В статье изучено поведение электрона в сильном кулоновском поле в $2+1$ измерениях в присутствии магнитного потока. Найдена волновая функция электрона в обрезанном кулоновском потенциале вблизи границы нижнего континуума энергий $-m$ и получены уравнения, определяющие спектр энергий электрона и критический заряд $a_{\mathrm{cr}}$. При $a>a_{\mathrm{cr}}$ низший уровень энергии электрона пересекает границу нижнего континуума энергий, а вакуум квантовой электродинамики в $2+1$ измерениях в сильном кулоновском поле перестраивается: в вакууме появляется новое 
состояние с энергией $E<-m$. Вакуум квантовой электродинамики при $a>a_{\mathrm{cr}}$ становится неустойчивым, что приводит к рождению позитрона, и одновременно вакуум приобретает отрицательный электрический заряд, который в точности равен заряду электрона. В $2+1$ измерениях критический заряд $a_{\mathrm{cr}}$ в присутствии магнитного потока явно зависит от спинового параметра $s$, так что при некоторых значениях $a_{\text {cr }}$ вакуум может также приобрести магнитный момент, равный спиновому магнитному моменту электрона. Показано, что вакуум квантовой электродинамики в $2+1$ измерениях в сильном кулоновском поле в присутствии положительного магнитного потока с $e_{0} B<0.8$ становится менее устойчивым по отношению к рождению позитронов со спином $s_{\mathrm{p}}=1$ и более устойчивым по отношению к рождению позитронов со спином $s_{\mathrm{p}}=-1$.

Благодарности. Работа поддержана совместным проектом РФФИ (грант № 06-02-89500-HHC_а) и Национального научного совета Тайваня (RFBR-NSC № 95WFD0400022) и частично Программой поддержки ведущих научных школ (грант НШ-3312.2008.2).

\section{Список литературы}

[1] Y. Aharonov, D. Bohm, Phys. Rev., 115:3 (1959), 485-491.

[2] M. Peshkin, A. Tonomura, The Aharonov-Bohm Effect, Lecture Notes in Phys., 340, Springer, Berlin, 1989.

[3] К. Хуанг, Кварки, лептоны и калибровочные поля, Мир, М., 1985.

[4] M. G. Alford, F. Wilczek, Phys. Rev. Lett., 62:10 (1989), 1071-1074.

[5] I. V. Tyutin, Electron scattering by a solenoid, arXiv: 0801.2167.

[6] V.R. Khalilov, Phys. Rev. A, 71:1 (2005), 012105.

[7] C. R. Hagen, Phys. Rev. Lett., 64:5 (1990), 503-506.

[8] V.R. Khalilov, C.-L. Ho, Ann. Physics, 323:5 (2008), 1280-1293; arXiv: 0708.3131.

[9] В. Р. Халилов, ТМФ, 149:3 (2006), 502-517.

[10] D. Porras, J. I. Cirac, Phys. Rev. Lett., 96 (2006), 250501.

[11] C.-L. Ho, V.R. Khalilov, Phys. Rev. A, 61:3 (2000), 032104.

[12] V.R. Khalilov, C.-L. Ho, Modern Phys. Lett. A, 13:8 (1998), 615-622.

[13] В. Р. Халилов, ТМФ, 116:2 (1998), 277-287.

[14] В.Б. Берестецкий, Е.М. Лифшиц, Л. П. Питаевский, Квантовая электродинамика, Наука, М., 1980.

[15] Дж. Д. Бьеркен, С. Д. Дрелл, Релятивистская квантовая теория. Т. I. Релятивистская квантовая механика, Наука, М., 1978.

[16] D. Cohen, Lecture notes in quantum mechanics, arXiv: quant-ph/0605180.

[17] W. Pieper, W. Greiner, Z. Phys., 218:4 (1969), 327-340.

[18] С. С. Герштейн, Я. Б. Зельдович, ЖЭТФ, 57 (1969), 654.

[19] Я. Б. Зельдович, В. С. Попов, УФН, 105:11 (1971), 403-440.

[20] А. Б. Мигдал, Фермионы и бозоны в силъных полях, Наука, М., 1978.

[21] J. Rafelski, L. P. Fulcher, A. Klein, Phys. Rep., 38:5 (1978), 227-361.

[22] M. Soffel, B. Müller, W. Greiner, Phys. Rep., 85:2 (1982), 51-122.

[23] А. А. Гриб, С. Г. Мамаев, В. М. Мостепаненко, Вакуумные квантовые эффекты в сильных полях, Энергоатомиздат, М., 1988.

[24] И. С. Градштейн, И.М. Рыжик, Таблицы интегралов, сумм, рядов и произведений, Физматлит, М., 1963. 